\title{
The Flat Central Configurations of Four Planet Motions $^{1)}$
}

\author{
He Shi Fengmei Zou ${ }^{2}$
}

\begin{abstract}
The flat central configurations of four planet motions is considered by using Wu Elimination (the Char-set method) in this paper. We obtain 12 collinear central configurations. Obtain a necessary condition for determining flat but non-collinear central configurations. And deduce that in general there are finitely many flat central configurations determined by their masses and their angular velocity.
\end{abstract}

Key Words: Central Configuration, Wu Elimination

\section{Introduction}

Wintner studied the configurations of planet motions in [1]. He conjectured that there are only finitely many central configurations with any number of particles of any masses (see [2]). For the case the particles are collinear, Moulton [3] obtained all solutions for arbitrary $n$ particles with distinct masses, the number of the solutions is $n ! / 2$. This result was reverified by Smale and his followers using topological methods $[4,5]$. Waldvogel $[6]$ showed that for any $n(n>4)$, there exist a group of $n$ particles which possess a non-flat central configuration. In [2], Professor Wu reduced the determination of the central configurations to a problem of polynomial equation-solving, and reverified the case of three particles by using the Char-set method (see [7]). In this paper, we determine the possible flat central configurations of four planets also by using the Wu Elimination (the Char-set method).

For the problem of determining flat central configurations of four bodies, from Wintner [1], it is known that for most sets of masses there exist a variety of (up to ten or more) central configurations; a square is a central configuration only in the case of the four equal masses; and for 4 given masses $m_{i}$, there exists at least one non-collinear flat central configuration only when the $m_{i}$ satisfy certain inequalities. In this paper, we give all the possible solutions of the collinear case; for the non-collinear case, we obtain that there are only finitely many solutions determined by the masses and the angular velocity.

\section{Notation and the Wintner Conjecture}

Let $m_{1}, m_{2}, \ldots, m_{n}$ be masses of $n$ particles moving under mutual Newtonian gravitational attractions, and $r_{1}, r_{2}, \ldots, r_{n}$ be the positions of these masses at a certain moment, with

\footnotetext{
${ }^{1}$ This work was supported in part by the NSF Grant CCR-9420857 and by the Chinese National Science Foundation.

${ }^{2)}$ Institute of Systems Science, Academia Sinica, Beijing 100080, P.R. China
} 
$r_{i} \neq r_{j}$ for $i \neq j$. A configuration formed of masses $m_{i}$ at position $r_{i}, i=1,2,3, \ldots, n$ is denoted by

$$
\left[\begin{array}{llll}
m_{1} & m_{2} & \ldots & m_{n} \\
r_{1} & r_{2} & \ldots & r_{n}
\end{array}\right]
$$

Definition 1. The center of $n$ particles $m_{1}, \ldots, m_{n}$ at the positions $r_{1}, \ldots, r_{n}$, is defined to be the position $\sum_{i=1}^{n} m_{i} r_{i} / \sum_{i=1}^{n} m_{i}$.

Definition 2. A configuration is called a central configuration with respect to the masses $m_{1}, m_{2}, \ldots, m_{n}$, if there are initial velocities of the masses $m_{i}$ such that under the Newtonian gravitational attractions the configurations formed by the masses during the motion will remain similar to the initial one.

From this definition, we know that a central configuration defines a class of configurations. As a direct consequence of Newtonian mechanics, for a central configuration the center of mass of the masses $m_{1}, m_{2}, \ldots m_{n}$ may be considered to be fixed during motion.

Definition 3. The inertial coordinate system associated to a central configuration is defined to be a cartesian coordinate system for which the origin is at the fixed center of mass of the masses $m_{1}, m_{2}, \ldots, m_{n}$.

In the following, we assume that $r_{1}, \ldots, r_{n}$ are the initial coordinates of the particles $m_{1}, \ldots, m_{n}$. By the definition of the center and the inertial coordinate system, we have

$$
\sum_{i=1}^{n} m_{i} r_{i}=0
$$

We set

$q_{3}(n):=$ number of central configurations with given masses $m_{1}, \ldots m_{n} ;$

$q_{2}(n):=$ number of central configurations with given masses $m_{1}, \ldots m_{n}$ for which the masses are in the same plane;

$q_{1}(n):=$ number of central configurations with given masses $m_{1}, \ldots m_{n}$ for which the masses are in the same line.

Wintner Conjecture: $q_{3}(n)$ and $q_{2}(n)$ are finite for all $n$ and masses $m_{1}, \ldots, m_{n}$.

\section{Reduction the Conjecture to Certain Polynomial-Solving}

Let $p i, i=1,2, \ldots, n$ be polynomials and $P S=(p 1, p 2, \ldots, p n)$ be a polynomial set. We denote the zero set of $P S$ by zero $(P S)$ and denote the restricted zero set of $P S$ by zerorc $(P S)$ for which the reality conditions are observed. We have to observe some reality conditions, for example, $r_{i j}, m_{i}(i, j=1,2, \ldots, n)>0$.

Taking planar inertial coordinate system $(x, y)$ with origin $\mathrm{O}$ at the center of masses. Let $r_{i}=\left(x_{i}, y_{i}\right), x_{i j}=x_{i}-x_{j}, y_{i j}=y_{i}-y_{j}, r_{i j}=\sqrt{\left(x_{i j}^{2}+y_{i j}^{2}\right)},(i, j=1,2, \ldots, n, i \neq j)$. By Newtonian mechanics, the Newtonian attraction of $m_{i}$ at position $r_{i}$ is equal to it's centrifugal force, hence we have a general system of equations (see [2])

$$
\begin{array}{ll}
\omega^{2} x_{i}=\sum_{j \neq i} m_{j} \frac{x_{i j}}{r_{i j}^{3}}, & i=1,2, \ldots, n, \\
\omega^{2} y_{i}=\sum_{j \neq i} m_{j} \frac{y_{i j}^{3}}{r_{i j}^{3}}, & i=1,2, \ldots, n
\end{array}
$$


where $\omega$ is an angular velocity and is a constant.

Let $u_{j}=x_{j}+\mathbf{i} y_{j}, v_{j}=x_{j}-\mathbf{i} y_{j}(j=1,2, \ldots, n)$, where $\mathbf{i}=\sqrt{-1}$, and $m_{0}=-\omega^{2}$, then the above equations become the following system of equations

$$
\begin{array}{ll}
\text { (I) } \quad m_{0} u_{i}+\sum_{j \neq i} \frac{m_{j}}{\left(v_{i}-v_{j}\right) r_{i j}}=0, \quad i=1,2, \ldots, n, \\
\text { (II) } \quad m_{0} v_{i}+\sum_{j \neq i} \frac{m_{j}}{\left(u_{i}-u_{j}\right) r_{i j}}=0, \quad i=1,2, \ldots, n,
\end{array}
$$

and

$$
\begin{array}{lll}
r_{i j}-\left(u_{i}-u_{j}\right)\left(v_{i}-v_{j}\right)=0, & i \neq j, & i, j=1,2, \ldots, n, \\
r_{i j}=r_{j i}, & i \neq j, & i, j=1,2, \ldots, n .
\end{array}
$$

Thus, the determination of flat central configurations is reduced to equation-solving with variables $u_{i}, v_{j}, r_{i j}$ as unknowns.

To simplify the notations we set $u_{i j}=u_{i}-u_{j}, v_{i j}=v_{i}-v_{j}, i<j$, and $u_{i j}=u_{1 j}-u_{1 i}, v_{i j}=$ $v_{1 j}-v_{1 i}, i \neq j, i, j=2, \ldots, n$.

\section{The case of $n=4$}

The problem of the central configurations of three bodies was studied in [2] by using the Char-set method. In this section we study the problem of four bodies. In this case, the equations (I) are the following

$$
\begin{aligned}
& q 1 \equiv m_{0} u_{1}+\frac{m_{2}}{\left(v_{1}-v_{2}\right) r_{12}}+\frac{m_{3}}{\left(v_{1}-v_{3}\right) r_{13}}+\frac{m_{4}}{\left(v_{1}-v_{4}\right) r_{14}}=0, \\
& q 2 \equiv m_{0} u_{2}-\frac{m_{1}}{\left(v_{1}-v_{1}\right) r_{12}}+\frac{m_{3}}{\left(v_{2}-v_{3}\right) r_{23}}+\frac{m_{4}}{\left(v_{2}-v_{4}\right) r_{24}}=0, \\
& q 3 \equiv m_{0} u_{3}-\frac{m_{1}}{\left(v_{1}-v_{3}\right) r_{13}}-\frac{m_{2}}{\left(v_{2}-v_{3}\right) r_{23}}+\frac{m_{4}}{\left(v_{3}-v_{4}\right) r_{34}}=0, \\
& q 4 \equiv m_{0} u_{4}-\frac{m_{1}}{\left(v_{1}-v_{4}\right) r_{14}}-\frac{m_{2}}{\left(v_{2}-v_{4}\right) r_{24}}-\frac{m_{3}}{\left(v_{3}-v_{4}\right) r_{34}}=0 .
\end{aligned}
$$

It is easy to see that this system is equivalent to the system which consist of $q 0 \equiv m_{1} u_{1}+$ $m_{2} u_{2}+m_{3} u_{3}+m_{4} u_{4}=0$ and any three from $q_{1}, q_{2}, q_{3}, q_{4}$.

Note that q0 is identical with any one of the following:

$$
\begin{aligned}
& q 01 \equiv\left(m_{1}+m_{2}+m_{3}+m_{4}\right) u_{1}-m_{2} u_{12}-m_{3} u_{13}-m_{4} u_{14}=0 \\
& q 02 \equiv\left(m_{1}+m_{2}+m_{3}+m_{4}\right) u_{2}+m_{1} u_{12}-m_{3} u_{23}-m_{4} u_{24}=0 \\
& q 03 \equiv\left(m_{1}+m_{2}+m_{3}+m_{4}\right) u_{3}+m_{1} u_{13}+m_{2} u_{23}-m_{4} u_{34}=0 \\
& q 04 \equiv\left(m_{1}+m_{2}+m_{3}+m_{4}\right) u_{4}+m_{1} u_{14}+m_{2} u_{24}+m_{4} u_{34}=0 .
\end{aligned}
$$

Clearing the fractions of $q 1, q 2, q 3, q 4$, we obtain $p 1, p 2, p 3, p 4$ respectively where

$$
\begin{aligned}
p 1 \equiv & v_{12} r_{12} v_{13} r_{13} v_{14} r_{14} m_{0} u_{1}+m_{2} v_{13} r_{13} v_{14} r_{14} \\
& +m_{3} v_{12} r_{12} v_{14} r_{14}+m_{4} v_{12} r_{12} v_{13} r_{13}=0, \\
p 2 \equiv & v_{12} r_{12} v_{23} r_{23} v_{24} r_{24} m_{0} u_{2}-m_{1} v_{23} r_{23} v_{24} r_{24} \\
& +m_{3} v_{12} r_{12} v_{24} r_{24}+m_{4} v_{12} r_{12} v_{23} r_{23}=0 \\
p 3 \equiv & v_{13} r_{13} v_{23} r_{23} v_{34} r_{34} m_{0} u_{3}-m_{1} v_{23} r_{23} v_{34} r_{34} \\
& -m_{2} v_{13} r_{13} v_{34} r_{34}+m_{4} v_{13} r_{13} v_{23} r_{23}=0 \\
p 4 \equiv & v_{14} r_{14} v_{24} r_{24} v_{34} r_{34} m_{0} u_{4}-m_{1} v_{24} r_{24} v_{34} r_{34} \\
& -m_{2} v_{14} r_{14} v_{34} r_{34}-m_{3} v_{14} r_{14} v_{24} r_{24}=0 .
\end{aligned}
$$


Let

$$
\begin{aligned}
& p 9 \equiv r_{12}^{2}-u_{12} v_{12}=0 \\
& p 10 \equiv r_{13}^{2}-u_{13} v_{13}=0 \\
& p 11 \equiv r_{14}^{2}-u_{14} v_{14}=0 \\
& p 12 \equiv r_{23}^{2}-u_{23} v_{23}=0 \\
& p 13 \equiv r_{24}^{2}-u_{24} v_{24}=0 \\
& p 14 \equiv r_{34}^{2}-u_{34} v_{34}=0 .
\end{aligned}
$$

For simplification, we introduce the notations $a=m_{1}+m_{2}+m_{3}+m_{4}$ and $f_{i j}=a+m_{0} r_{13}^{3}, i<$ $j, \quad i, j=1,2,3,4$.

Now we consider the system of polynomials

$$
\{q 01, p 1, p 2, p 4, p 9, p 10, p 11, p 12, p 13, p 14\} \text {. }
$$

After eliminating variables $u_{1}, u_{12}, u_{13}, u_{14}$ of $p 1$ step by step via pseudo-devision by polynomials $[q 01, p 9, p 10, p 11]$, we obtain a polynomial

$$
p 15 \equiv m_{2} r_{13} r_{14} v_{13} v_{14} f_{12}+m_{3} r_{12} r_{14} v_{12} v_{14} f_{13}+m_{4} r_{12} r_{13} v_{12} v_{13} f_{14}=0 .
$$

For polynomials $p 2,(p 4)$, after eliminating the variables $u_{2}, u_{12}, u_{23}, u_{24},\left(u_{4}, u_{14}, u_{24}, u_{34}\right.$, respectively) by polynomials $[q 02, p 9, p 12, p 13]([q 04, p 11, p 13, p 14]$, respectively) and replacing $v_{i j}(i, j \neq 1)$ with $\left(v_{1 j}-v_{1 i}\right)$ in both of the polynomials we obtain

$$
\begin{aligned}
q 16 \equiv & m_{1} r_{23} r_{24}\left(v_{13}-v_{12}\right)\left(v_{14}-v_{12}\right) f_{12}-m_{3} r_{12} r_{24} v_{12}\left(v_{14}-v_{12}\right) f_{23} \\
& -m_{4} r_{12} r_{23} v_{12}\left(v_{13}-v_{12}\right) f_{24}=0, \\
q 18 \equiv & m_{1} r_{24} r_{34}\left(v_{14}-v_{12}\right)\left(v_{14}-v_{13}\right) f_{14}+m_{2} r_{14} r_{34} v_{14}\left(v_{14}-v_{13}\right) f_{24} \\
& +m_{3} r_{14} r_{24} v_{14}\left(v_{14}-v_{12}\right) f_{34}=0 .
\end{aligned}
$$

For the system (II), we have the similar results

$$
\begin{aligned}
& q q 0 \equiv m_{1} v_{1}+m_{2} v_{2}+m_{3} v_{3}+m_{4} v_{4}=0, \\
& q 5 \equiv m_{0} v_{1}+\frac{m_{2}}{\left(u_{1}-u_{2}\right) r_{12}}+\frac{m_{3}}{\left(u_{1}-u_{3}\right) r_{13}}+\frac{m_{4}}{\left(u_{1}-u_{4}\right) r_{14}}=0, \\
& q 6 \equiv m_{0} v_{2}-\frac{m_{1}}{\left(u_{1}-u_{2}\right) r_{12}}+\frac{m_{3}}{\left(u_{2}-u_{3}\right) r_{23}}+\frac{m_{4}}{\left(u_{2}-u_{4}\right) r_{24}}=0, \\
& q 7 \equiv m_{0} v_{3}-\frac{m_{1}}{\left(u_{1}-u_{3}\right) r_{13}}-\frac{m_{2}}{\left(u_{2}-u_{3}\right) r_{23}}+\frac{m_{4}}{\left(u_{3}-u_{4}\right) r_{34}}=0, \\
& q 8 \equiv m_{0} v_{4}-\frac{m_{1}}{\left(u_{1}-u_{4}\right) r_{14}}-\frac{m_{2}}{\left(u_{2}-u_{4}\right) r_{24}}-\frac{m_{3}}{\left(u_{3}-u_{4}\right) r_{34}}=0 .
\end{aligned}
$$

Clearing the fractions, we have the following polynomial equations

$$
\begin{aligned}
p 5 \equiv & u_{12} r_{12} u_{13} r_{13} u_{14} r_{14} m_{0} v_{1}+m_{2} u_{13} r_{13} u_{14} r_{14}+m_{3} u_{12} r_{12} u_{14} r_{14} \\
& +m_{4} u_{12} r_{12} u_{13} r_{13}=0 \\
p 6 \equiv & u_{12} r_{12} u_{23} r_{23} u_{24} r_{24} m_{0} v_{2}-m_{1} u_{23} r_{23} u_{24} r_{24}+m_{3} u_{12} r_{12} u_{24} r_{24}+ \\
& m_{4} u_{12} r_{12} u_{23} r_{23}=0 \\
p 7 \equiv & u_{13} r_{13} u_{23} r_{23} u_{34} r_{34} m_{0} v_{3}-m_{1} u_{23} r_{23} u_{34} r_{34}-m_{2} u_{13} r_{13} u_{34} r_{34} \\
& +m_{4} u_{13} r_{13} u_{23} r_{23}=0, \\
p 8 \equiv & u_{14} r_{14} u_{24} r_{24} u_{34} r_{34} m_{0} v_{4}-m_{1} u_{24} r_{24} u_{34} r_{34}-m_{2} u_{14} r_{14} u_{34} r_{34} \\
& -m_{3} u_{14} r_{14} u_{24} r_{24}=0 .
\end{aligned}
$$


Similarly to the equations $p 1, p 2, p 3, p 4$, we can obtain polynomial equations $p 19, p 20, p 21$ from $p 5, p 6, p 8$ respectively where

$$
\begin{aligned}
p 19 \equiv & m_{2} r_{13} r_{14} u_{13} u_{14} f_{12}+m_{3} r_{12} r_{14} u_{12} u_{14} f_{13} \\
& +m_{4} r_{12} r_{13} u_{12} u_{13} f_{14}=0 \\
p 20 \equiv & m_{1} r_{23} r_{24}\left(u_{13}-u_{12}\right)\left(u_{14}-u_{12}\right) f_{12}-m_{3} r_{12} r_{24} u_{12}\left(u_{14}-u_{12}\right) f_{23} \\
& -m_{4} r_{12} r_{23} u_{12}\left(u_{13}-u_{12}\right) f_{24}=0, \\
p 21 \equiv & m_{1} r_{24} r_{34}\left(u_{14}-u_{12}\right)\left(u_{14}-u_{13}\right) f_{14}+m_{2} r_{14} r_{34} u_{14}\left(u_{14}-u_{13}\right) f_{24} \\
& +m_{3} r_{14} r_{24} u_{14}\left(u_{14}-u_{12}\right) f_{34}=0 .
\end{aligned}
$$

And we have $q q 01 \equiv\left(m_{1}+m_{2}+m_{3}+m_{4}\right) v_{1}-m_{2} v_{12}-m_{3} v_{13}-m_{4} v_{14}=0$. Since while doing the pseudo-dividing from $p 1, p 2, p 4, p 5, p 6, p 8$ to $p 15, p 16, p 18, p 19, p 20, p 21$, all the initials of the middle polynomials are not zero, we have

$$
\begin{aligned}
z \operatorname{ero}\{p 1, p 2, \ldots, p 14\}= & \operatorname{zero}\{q 01, p 1, p 2, p 4, p 9, p 10, p 11, p 12, p 13, p 14\} \\
& \cap \operatorname{erro}\{q q 01, p 5, p 6, p 8, p 9, p 10, p 11, p 12, p 13, p 14\},
\end{aligned}
$$

and

$$
\begin{aligned}
\text { zero }_{r c}\{q 01, p 1, & p 2, p 4, p 9, p 10, p 11, p 12, p 13, p 14\}= \\
& \text { zerorc }\{q 01, p 15, p 16, p 18, p 9, p 10, p 11, p 12, p 13, p 14\}, \\
\text { zero }_{r c}\{q q 01, p 5, & p 6, p 8, p 9, p 10, p 11, p 12, p 13, p 14\}= \\
& \text { zerorc }\{q q 01, p 19, p 20, p 21, p 9, p 10, p 11, p 12, p 13, p 14\} .
\end{aligned}
$$

To solve system $\{q 01, p 15, p 16, p 18, p 9, p 10, p 11, p 12, p 13, p 14\}$, we set the following order

$$
\begin{aligned}
m_{1} \prec m_{2} \prec m_{3} & \prec m_{4} \prec r_{12} \prec r_{13} \prec r_{14} \prec r_{23} \prec r_{24} \prec r_{34} \prec m_{0} \prec f_{12} \prec f_{13} \\
& \prec f_{14} \prec f_{23} \prec f_{24} \prec f_{34} \prec v_{14} \prec v_{13} \prec v_{12} \prec u_{1}
\end{aligned}
$$

By using Wu elimination (Char-set method), we decompose the system $\{q 01, q 15, q 16, q 18\}$ and obtain five systems of polynomial equations as follows:

(1). $Z S 1=z \operatorname{ero}\{z 11, z 12, z 13, z 14, z 15, z 16\}$, in which

$$
\begin{aligned}
& z 11 \equiv a u_{1}-m_{2} u_{12}-m_{3} u_{13}-m_{4} u_{14}=0, \\
& z 12 \equiv f_{12}=0, \\
& z 13 \equiv m_{3} r_{14} v_{14} f_{13}+m_{4} r_{13} v_{13} f_{14}=0, \\
& z 14 \equiv f_{24}=0, \\
& z 15 \equiv f_{23}=0, \\
& z 16 \equiv m_{4} r_{13} m_{1} r_{34} f_{14}+m_{4} r_{13} m_{3} r_{14} f_{34}+m_{3} r_{14} f_{13} m_{1} r_{34}=0 .
\end{aligned}
$$

(2). $Z S 2=z \operatorname{ero}\{z 21, z 22, z 23, z 24, z 25\}$, in which $z 21=z 11, z 22=z 12, z 23=z 13$ and

$$
\begin{aligned}
z 24 \equiv & -v_{12} m_{3} r_{24} f_{23}-v_{12} f_{24} r_{23} m_{4}+m_{3} r_{24} f_{23} v_{14}+v_{13} f_{24} r_{23} m_{4}=0, \\
z 25 \equiv & r_{13} r_{23} m_{4} m_{2} r_{14} r_{34} f_{24}+r_{13} r_{23} m_{4} m_{1} r_{24} r_{34} f_{14}+r_{13} m_{3} r_{24} f_{23} m_{2} r_{14} r_{34} \\
& +r_{13} r_{23} m_{4} m_{3} r_{14} r_{24} f_{34}+m_{3} r_{14} f_{13} r_{23} m_{1} r_{24} r_{34}=0 .
\end{aligned}
$$

(3). $Z S 3=z \operatorname{ero}\{z 31, z 32, z 33, z 34, z 35, z 36\}$, in which $z 31=z 11, z 32=z 14$ and

$$
\begin{aligned}
z 33 \equiv & v_{12} m_{1} r_{23} f_{12}+v_{12} f_{23} r_{12} m_{3}-m_{1} r_{23} f_{12} v_{13}=0 \\
z 34 \equiv & -m_{1} r_{34} f_{14} v_{14}+m_{1} r_{34} f_{14} v_{13}-m_{3} r_{14} v_{14} f_{34}=0 \\
z 35 \equiv & m_{1} r_{34} f_{14} m_{3} r_{12} r_{14} f_{13}+m_{3} r_{14} f_{34} m_{4} r_{12} r_{13} f_{14}+m_{3} r_{14}^{2} f_{34} r_{13} m_{2} f_{12} \\
& +m_{1} r_{34} f_{14}^{2} m_{4} r_{12} r_{13}+m_{1} r_{34} f_{14} r_{13} m_{2} r_{14} f_{12}=0 \\
z 36 \equiv & r_{34} m_{2} r_{13} r_{14} m_{1} r_{23} f_{12}+r_{34} m_{2} r_{13} r_{14} m_{3} r_{12} f_{23}+r_{34} r_{12} m_{3} f_{13} m_{1} r_{23} r_{14} \\
& +m_{4} r_{12} r_{23} r_{13} m_{3} r_{14} f_{34}+m_{4} r_{12} r_{23} r_{13} m_{1} r_{34} f_{14}=0
\end{aligned}
$$


(4). $Z S 4=z \operatorname{ero}\{z 41, z 42, z 43, z 44, z 45, z 46\}$, in which $z 41=z 11, z 42=z 12$ and

$$
\begin{aligned}
& z 43 \equiv f_{13}=0, \\
& z 44 \equiv f_{14}=0, \\
& z 45 \equiv m_{2} m_{4} r_{23} r_{34} f_{24}+m_{2} m_{3} r_{24} r_{34} f_{23}+m_{3} m_{4} r_{23} r_{24} f_{34}=0, \\
& z 46 \equiv m_{3} r_{24} f_{34} v_{12}+m_{2} r_{34} f_{24} v_{13}-\left(m_{2} r_{34} f_{24}+m_{3} r_{24} f_{34}\right) v_{14}=0 .
\end{aligned}
$$

(5). $Z S 5=z \operatorname{ero}\{z 51, z 52, z 53, z 54\}$, in which $z 51=z 11$ and

$$
\begin{aligned}
z 52 \equiv & -m_{2} r_{13} r_{14} v_{13} v_{14} f_{12}-m_{3} r_{12} r_{14} v_{12} v_{14} f_{13}-m_{4} r_{12} r_{13} v_{12} v_{13} f_{14}=0, \\
z 53 \equiv & -v_{14}^{2} m_{3} r_{12} r_{14} f_{13} m_{1} r_{24} r_{34} f_{14}-v_{14}^{2} m_{3} r_{12} r_{14}^{2} f_{13} m_{2} r_{34} f_{24} \\
& -v_{14}^{2} m_{3}^{2} r_{12} r_{14}^{2} f_{13} r_{24} f_{34}+v_{14} m_{3} r_{12} r_{14} f_{13} m_{1} r_{24} r_{34} f_{14} v_{13} \\
& -v_{14} m_{2} r_{13} r_{14}^{2} v_{13} f_{12} m_{3} r_{24} f_{34}-v_{14} m_{4} r_{12} r_{13} v_{13} f_{14}^{2} m_{1} r_{24} r_{34} \\
& -v_{14} m_{2} r_{13} r_{14} v_{13} f_{12} m_{1} r_{24} r_{34} f_{14}+v_{14} m_{3} r_{12} r_{14}^{2} f_{13} m_{2} r_{34} f_{24} v_{13} \\
& -v_{14} m_{4} r_{12} r_{13} v_{13} f_{14} m_{3} r_{14} r_{24} f_{34}-v_{14} m_{4} r_{12} r_{13} v_{13} f_{14} m_{2} r_{14} r_{34} f_{24} \\
& +m_{4} r_{12} r_{13} v_{13}^{2} f_{14} m_{2} r_{14} r_{34} f_{24}+m_{4} r_{12} r_{13} v_{13}^{2} f_{14}^{2} m_{1} r_{24} r_{34} \\
& +m_{2} r_{13} r_{14} v_{13}^{2} f_{12} m_{1} r_{24} r_{34} f_{14}=0, \\
z 54 \equiv & m_{2} r_{13} r_{14} r_{34} m_{1} r_{23} r_{24} f_{12}+m_{2} r_{13} r_{14} r_{34} m_{4} r_{12} r_{23} f_{24} \\
& +m_{2} r_{13} r_{14} r_{34} m_{3} r_{12} r_{24} f_{23}+r_{13} m_{4} r_{12} r_{23} m_{3} r_{24} f_{34} r_{14} \\
& +r_{13} m_{4} r_{12} r_{23} m_{1} r_{24} r_{34} f_{14}+r_{34} m_{3} r_{12} f_{13} m_{1} r_{23} r_{24} r_{14}=0 .
\end{aligned}
$$

Similarly decomposing the system $\{q q 01, p 19, p 20, p 21\}$ we also obtain five systems of polynomial equations. They may be got from above via replacing the $v_{1}, u_{i j}$ with $u_{1}, v_{i j}$, respectively.

Combining these two groups of systems of polynomial equations and adding the polynomials $p 9, p 10, p 11, p 12, p 13, p 14$, we get 15 systems of polynomial equations. We solve these systems by using the Char-set method and find that all the possible solutions of each system are divided into three cases according to how many $f_{i j}=0$ as following:

Case 1, Only one $f_{i j}$ equals to zero. It deduces to the possible solutions of collinear case;

Case 2, Two $f_{i j}$ equal to zero. Then it is easy to see that there are at least three $f_{i j}$ are zero. Considering the reality conditions, it has no solutions.

Case 3 , There is not any $f_{i j}$ equal to zero. It leads to the possible solutions of non-collinear case.

Most of the 15 systems deduce to the case 2 which get the solutions either all $r_{i j}=0$ or three of the four points are both collinear and co-circle.

\subsection{Collinear case}

In the case 1 , we have the following system of polynomial equations

$$
E Q S 1=\{z 21, z 22, z 23, z 24, z 25, y 11, y 12, y 13, p 9, p 10, p 11, p 12, p 13, p 14\},
$$

in which $z 21, z 22, z 23, z 24, z 25$ are given in the system $Z S 2$ and $p 9, p 10, \cdots, p 14$ are given at the page 4 and $y 11, y 12, y 13$ are given by the $z 21, z 24, z 25$ via replacing the $v_{1}, u_{i j}$ with $u_{1}, v_{i j}, i, j=1,2,3,4$. respectively.

Note that the total degree of some polynomials in the system $E Q S 1$ is 7 . So, it is very complex to solve EQ1. However, we can get the characteristic sets of EQS1 by using Wu Elimination. We set the order as following 


$$
\begin{gathered}
m_{1} \prec m_{2} \prec m_{3} \prec m_{4} \prec r_{12} \prec r_{13} \prec r_{23} \prec r_{24} \prec r_{34} \prec m_{0} \prec f_{12} \prec f_{13} \\
\prec f_{14} \prec f_{23} \prec f_{24} \prec f_{34} \prec u_{14} \prec u_{13} \prec u_{12} \prec v_{14} \prec v_{13} \prec v_{12} \prec u_{1} \prec v_{1} .
\end{gathered}
$$

We obtain 12 characteristic sets of EQS1 They correspond to the 12 possible collinear central configurations. For example, we have the following one of the 12 characteristic sets:

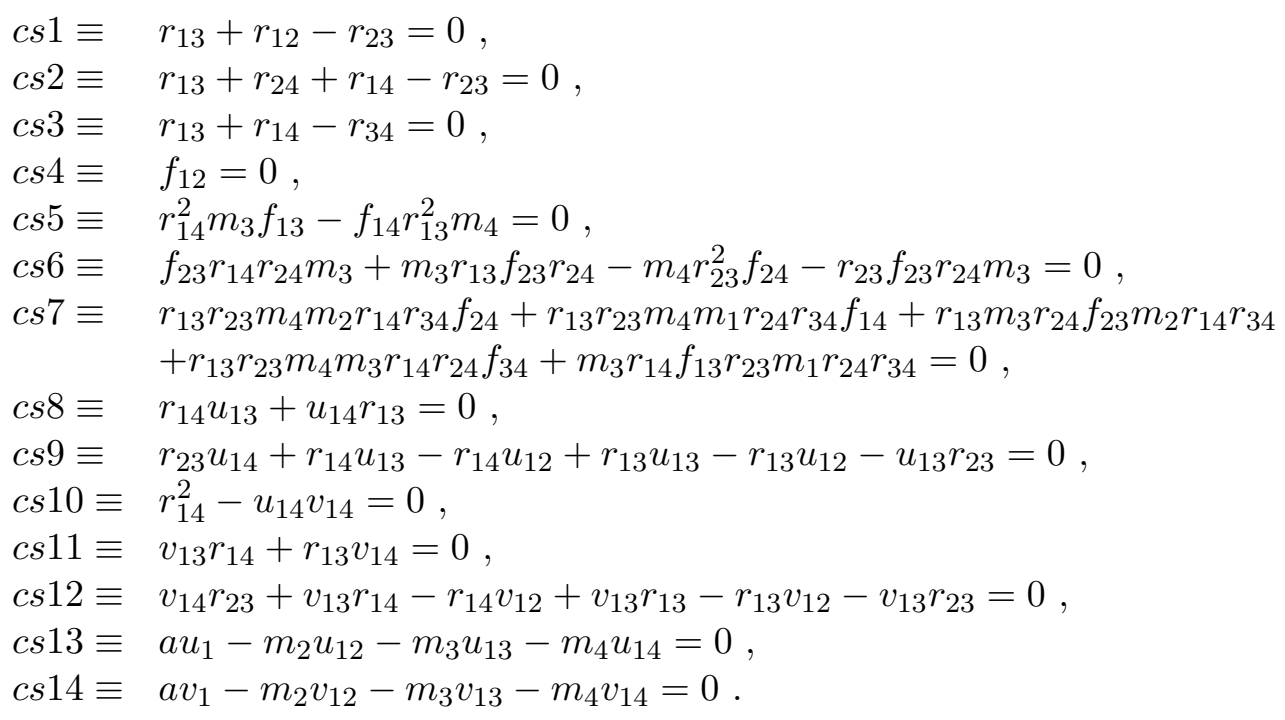

Hence, we have the following

Theorem 1. There are 12 collinear central configurations for four planets.

\subsection{Flat but non-collinear case}

In case 3 , we have the following system of polynomial equations:

$$
E Q S 2=\{z 51, z 52, z 53, z 54, y 21, y 22, y 23, y 24, p 9, p 10, p 11, p 12, p 13, p 14\}
$$

in which $z 51, z 52, z 53, z 54$ are given in the system $Z S 5$ and $p 9, p 10, \cdots, p 14$ as above in the system $E Q S 1$ and $y 21, y 22, y 23, y 24$ are given by the $z 51, z 52, z 53, z 54$ via replacing $v_{1}, u_{i j}$ with $u_{1}, v_{i j}$ respectively.

We set the following order

$$
\begin{aligned}
& m_{1} \prec m_{2} \prec m_{3} \prec m_{4} \prec r_{12} \prec r_{13} \prec r_{23} \prec r_{24} \prec r_{34} \prec m_{0} \prec g_{12} \prec g_{13} \\
& \prec g_{14} \prec g_{23} \prec g_{24} \prec g_{34} \prec u_{14} \prec u_{13} \prec u_{12} \prec v_{14} \prec v_{13} \prec v_{12} \prec u_{1} \prec v_{1}
\end{aligned}
$$

and by using Wu Elimination we obtain the following character set which has possible real 
solutions.

$$
\begin{aligned}
& c s 21 \equiv r_{12}^{4} r_{34}^{2}-r_{23}^{2} r_{12}^{2} r_{34}^{2}+r_{23}^{2} r_{12}^{2} r_{13}^{2}-r_{12}^{2} r_{24}^{2} r_{34}^{2}-r_{13}^{2} r_{12}^{2} r_{34}^{2}+r_{14}^{2} r_{12}^{2} r_{24}^{2} \\
& +r_{12}^{2} r_{34}^{4}-r_{12}^{2} r_{34}^{2} r_{14}^{2}-r_{12}^{2} r_{13}^{2} r_{24}^{2}-r_{14}^{2} r_{23}^{2} r_{12}^{2}+r_{14}^{4} r_{23}^{2}+r_{14}^{2} r_{23}^{4} \\
& +r_{13}^{2} r_{34}^{2} r_{14}^{2}-r_{14}^{2} r_{34}^{2} r_{23}^{2}+r_{23}^{2} r_{24}^{2} r_{34}^{2}-r_{14}^{2} r_{23}^{2} r_{24}^{2}+r_{13}^{2} r_{24}^{4}+r_{13}^{4} r_{24}^{2} \\
& -r_{13}^{2} r_{23}^{2} r_{24}^{2}-r_{13}^{2} r_{14}^{2} r_{24}^{2}-r_{13}^{2} r_{14}^{2} r_{23}^{2}-r_{13}^{2} r_{24}^{2} r_{34}^{2}=0 \text {, } \\
& c s 22 \equiv 2 r_{12}^{2} r_{13}^{2} r_{14}^{2} g_{13}-2 g_{12} r_{13}^{2} r_{14}^{2} r_{23}^{2}+r_{13}^{2} g_{12} r_{14}^{2} r_{12}^{2}+r_{13}^{4} g_{12} r_{14}^{2} \\
& -r_{12}^{2} g_{13} r_{34}^{4}+2 r_{12}^{2} r_{13}^{2} g_{13} r_{34}^{2}+2 r_{12}^{2} g_{13} r_{34}^{2} r_{14}^{2}+g_{12} r_{13}^{2} r_{12}^{2} r_{34}^{2} \\
& -g_{12} r_{13}^{4} r_{12}^{2}+r_{13}^{2} g_{12} r_{14}^{2} r_{34}^{2}-r_{13}^{2} g_{12} r_{14}^{4}-g_{12} r_{13}^{2} r_{24}^{2} r_{34}^{2} \\
& +r_{13}^{4} g_{12} r_{24}^{2}+r_{13}^{2} g_{12} r_{24}^{2} r_{14}^{2}-r_{12}^{2} r_{13}^{4} g_{13}-r_{14}^{4} r_{12}^{2} g_{13}=0 \text {, } \\
& c s 23 \equiv 2 r_{12}^{2} g_{14} r_{13}^{2}-r_{12}^{2} g_{13} r_{34}^{2}+r_{12}^{2} r_{13}^{2} g_{12}+r_{14}^{2} g_{12} r_{13}^{2} \\
& -g_{12} r_{13}^{2} r_{24}^{2}+r_{12}^{2} g_{13} r_{13}^{2}+r_{14}^{2} r_{12}^{2} g_{13}=0 \text {, } \\
& c s 24 \equiv r_{13}^{2} r_{12}^{2} g_{14}^{2}-g_{13} r_{14}^{2} g_{23} r_{12}^{2}-g_{23} r_{12}^{2} g_{14} r_{13}^{2}-r_{14}^{2} g_{23} g_{12} r_{13}^{2} \\
& -r_{14}^{2} r_{12}^{2} g_{13}^{2}-r_{14}^{2} g_{13} g_{12} r_{13}^{2}-r_{14}^{2} r_{12}^{2} g_{13} g_{12}-r_{14}^{2} r_{13}^{2} g_{12}^{2}=0 \text {, } \\
& c s 25 \equiv r_{13}^{2} g_{12} g_{14}+r_{13}^{2} g_{14}^{2}+r_{13}^{2} g_{14} g_{24}-r_{14}^{2} g_{13} g_{23}-r_{14}^{2} g_{13}^{2}-r_{14}^{2} g_{13} g_{12}=0 \text {, } \\
& c s 26 \equiv g_{34}+g_{24}+g_{23}+g_{14}+g_{13}+g_{12}=0 \text {, } \\
& c s 27 \equiv r_{14}^{2} r_{13}^{2} g_{12}^{2} u_{13} u_{14}-u_{14} r_{13}^{2} r_{12}^{2} g_{14}^{2} u_{13}-u_{14}^{2} r_{13}^{2} r_{12}^{2} g_{14} g_{13} \\
& -r_{14}^{2} u_{13}^{2} r_{12}^{2} g_{13} g_{14}-r_{14}^{2} u_{13} r_{12}^{2} g_{13}^{2} u_{14}=0 \text {, } \\
& c s 28 \equiv u_{14} r_{13}^{2} r_{12}^{2} g_{14}+r_{14}^{2} u_{13} r_{12}^{2} g_{13}+r_{14}^{2} r_{13}^{2} u_{12} g_{12}=0 \text { ， } \\
& c s 29 \equiv r_{14}^{2}-u_{14} v_{14}=0 \text {, } \\
& c s 30 \equiv r_{13}^{2}-u_{13} v_{13}=0, \\
& c s 31 \equiv r_{12}^{2}-u_{12} v_{12}=0, \\
& c s 32 \equiv a u_{1}-m_{2} u_{12}-m_{3} u_{13}-m_{4} u_{14}=0, \\
& c s 33 \equiv a v_{1}-m_{2} v_{12}-m_{3} v_{13}-m_{4} v_{14}=0,
\end{aligned}
$$

in which the notations $g_{12}, g_{13}, g_{14}, g_{23}, g_{24}, g_{34}$ are introduced by the following equations

$$
\begin{aligned}
& h 1 \equiv m_{1} m_{2} f_{12}-r_{12} g_{12}=0, \\
& h 2 \equiv m_{1} m_{3} f_{13}-r_{13} g_{13}=0, \\
& h 3 \equiv m_{1} m_{4} f_{14}-r_{14} g_{14}=0, \\
& h 4 \equiv m_{2} m_{3} f_{23}-r_{23} g_{23}=0, \\
& h 5 \equiv m_{2} m_{4} f_{24}-r_{24} g_{24}=0, \\
& h 6 \equiv m_{3} m_{4} f_{34}-r_{34} g_{34}=0 .
\end{aligned}
$$

Hence, from this characteristic set we know that all the solutions are uniquely determined by $m_{i}, m_{0}$, or $m_{i}, \omega$. So there are finitely many solutions of the system $E Q S 2$ which are determined by $m_{i}, m_{0}$, or $m_{i}, \omega$. Thus we have

Theorem 2. In general, the number of the central configuration in planet motions of 4 bodies is finite.

\section{Reference}

1. A. Wintner, The Analytical Foundations of Celestial Mechanics, Second Printing, 1947.

2. Wu Wen-tsun, Central Configurations in Planet Motions and Vortex Motions, MMRes. Preprints No. 13(1995) 1-14. 
3. F. R. Moulton, The Straight Line Solutions for the Problem of n-bodies, Annals of Math., 12(1910) 1-17.

4. S. Smale, Topology and Mechanics, I, II. Invent. Math. 10(1970) 305-331; 11(1970) 45-64.

5. S. Smale, Problems on the Nature of Relative Equilibria in Celestial Mechanics, in Manifolds-Amsterdam 1970, 194-198.

6. J. Waldvogel, Note Concerning a Conjecture by A. Wintner, Celestial Mechanics, $5(1972) 37-40$.

7. Wu Wen-tsun, Basic Principles of Mechanical Theorem Proving in Geometries, Volume I: Part of Elementary Geometries, Science Press, Beijing(in Chinese), 1984. 